\title{
SOME RESULTS OF THE MARIÑO-VAFA FORMULA
}

\author{
YI LI
}

\begin{abstract}
In this paper we derive some new Hodge integral identities by taking the limits of Mariño-Vafa formula. These identities include the formula of $\lambda_{1} \lambda_{g}$-integral on $\overline{\mathcal{M}}_{g, 1}$, the vanishing result of $\lambda_{g} \mathrm{ch}_{2 l}(\mathbb{E})$-integral on $\overline{\mathcal{M}}_{g, 1}$ for $1 \leq l \leq g-3$. Using the differential equation of Hodge integrals, we give a recursion formula of $\lambda_{g-1}$-integrals. Finally, we give two simple proofs of $\lambda_{g}$ conjecture and some examples of low genus integral.
\end{abstract}

\section{Introduction}

Based on string duality, Mariño and Vafa [10] conjectured a closed formula on certain Hodge integrals in terms of representations of symmetric groups. Recently, C.C. Liu, K. Liu and J. Zhou [6] proved this formula and derived some consequences from it [7]. In this paper we follow their method to derive some new Hodge integral identities. One of the main results of this paper is the following identity: if $1 \leq m \leq$ $2 g-3$, then

$$
\begin{aligned}
& -(2 g-2-m) !(-1)^{2 g-3-m} \int_{\overline{\mathcal{M}}_{g, 1}} \lambda_{g} \mathrm{ch}_{2 g-2-m}(\mathbb{E}) \psi_{1}^{m} \\
= & b_{g} \sum_{k=0}^{m-1} \frac{(-1)^{2 g-1-k}\left(\begin{array}{c}
2 g-1 \\
k
\end{array}\right)\left(\begin{array}{c}
2 g-1-k \\
2 g-1-m
\end{array}\right)}{2 g-1-k} B_{2 g-1-m} \\
+ & \frac{1}{2} \sum_{g_{1}+g_{2}=g, g_{1}, g_{2}>0} b_{g_{1}} b_{g_{2}} \sum_{k=0}^{\min \left(2 g_{2}-1, m-1\right)} \frac{(-1)^{2 g_{2}-1-k}\left(\begin{array}{c}
2 g_{2}-1 \\
k
\end{array}\right)\left(\begin{array}{c}
2 g-1-k \\
2 g-1-m
\end{array}\right)}{2 g-1-k} B_{2 g-1-m} .
\end{aligned}
$$

As a consequence, we find a new Hodge integral identity: if $g \geq 2$, then

$$
\int_{\overline{\mathcal{M}}_{g, 1}} \lambda_{1} \lambda_{g} \psi_{1}^{2 g-3}=\frac{1}{12}\left[g(2 g-3) b_{g}+b_{1} b_{g-1}\right],
$$

and also a vanishing result: if $g \geq 2$, then for any $1 \leq t \leq g-1$, we have

$$
\int_{\overline{\mathcal{M}}_{g, 1}} \lambda_{g} \operatorname{ch}_{2 t}(\mathbb{E}) \psi_{1}^{2(g-1-t)}=0 .
$$

Recently, Liu-Xu [8] derived a generalized formula for Hodge integrals of type (2) by using the $\lambda_{g}$ conjecture.

The rest of this paper is organized as follows: In Section 2, we recall the MariñoVafa formula and the Mumford's relations. In Section 3, we prove our main theorem and derive a new Hodge integral identity. In Section 4, we give another simple proof

Received by the editors April 20, 2006. 
of $\lambda_{g}$ conjecture. In Section 5 , we derive a recursion formula of $\lambda_{g-1}$-integrals. In the last section, we list some low genus examples.

\section{Preliminaries}

2.1. Partitions. A partition $\mu$ of a positive integer $d$ is a sequence of integers $\mu_{1} \geq$ $\mu_{2} \geq \cdots \geq \mu_{l(\mu)}>0$ such that

$$
\mu_{1}+\cdots+\mu_{l(\mu)}=d=|\mu|,
$$

for each positive integer $i$, let

$$
m_{i}(\mu)=\left|\left\{j \mid \mu_{j}=i, 1 \leq j \leq l(\mu)\right\}\right| .
$$

The automorphism group $\operatorname{Aut}(\mu)$ of $\mu$ consists of possible permutations among the $\mu_{i}$ 's, hence its order is given by

$$
|\operatorname{Aut}(\mu)|=\prod_{i} m_{i}(\mu) !
$$

define the numbers

$$
\kappa_{\mu}=\sum_{i=1}^{l(\mu)} \mu_{i}\left(\mu_{i}-2 i+1\right), \quad z_{\mu}=\prod_{j} m_{j}(\mu) ! j^{m_{j}(\mu)} .
$$

The Young diagram of $\mu$ has $l(\mu)$ rows of adjacent squares: the $i$-th row has $\mu_{i}$ squares. The diagram of $\mu$ can be defines as the set of points $(i, j) \in \mathbb{Z} \times \mathbb{Z}$ such that $1 \leq j \leq \mu_{i}$, the conjugate of a partition $\mu$ is the partition $\mu^{\prime}$ whose diagram is the transpose of the diagram $\mu$. Finally, we introduce the hook length of $\mu$ at the squre $x \in(i, j)$ :

$$
h(x)=\mu_{i}+\mu_{j}^{\prime}-i-j+1 .
$$

Each partition $\mu$ of $d$ corresponds to a conjugacy class $C(\mu)$ of the symmetric group $S_{d}$ and each partition $\nu$ corresponds to an irreducible representation $R_{\nu}$ of $S_{d}$, let $\chi_{\nu}(C(\mu))=\chi_{R_{\nu}}(C(\mu))$ be the value of the character $\chi_{R_{\nu}}$ on the conjugacy class $C(\mu)$.

2.2. Mariño-Vafa formula. Let $\overline{\mathcal{M}}_{g, n}$ denote the Deligne-Mumford moduli stack of stable curves of genus $g$ with $n$ marked points. Let $\pi: \overline{\mathcal{M}}_{g, n+1} \rightarrow \overline{\mathcal{M}}_{g, n}$ be the universal curve, and let $\omega_{\pi}$ be the relative dualizing sheaf. The Hodge bundle

$$
\mathbb{E}=\pi_{*} \omega_{\pi}
$$

is a rank $g$ vector bundle over $\overline{\mathcal{M}}_{g, n}$ whose fiber over $\left[\left(C, x_{1}, \cdots, x_{n}\right)\right] \in \overline{\mathcal{M}}_{g, n}$ is $H^{0}\left(C, \omega_{C}\right)$, the complex vector space of holomorphic one forms on $C$. Let $s_{i}: \overline{\mathcal{M}}_{g, n} \rightarrow$ $\overline{\mathcal{M}}_{g, n+1}$ denote the section of $\pi$ which corresponds to the $i$-th marked point, and let

$$
\mathbb{L}_{i}=s_{i}^{*} \omega_{\pi}
$$

be the line bundle over $\overline{\mathcal{M}}_{g, n}$ whose fiber over $\left[\left(C, x_{1}, \cdots, x_{n}\right)\right] \in \overline{\mathcal{M}}_{g, n}$ is the cotangent line $T_{x_{i}}^{*} C$ at the $i$-th marked point $x_{i}$. Consider the Hodge integral

$$
\int_{\overline{\mathcal{M}}_{g, n}} \psi_{1}^{j_{1}} \cdots \psi_{n}^{j_{n}} \lambda_{1}^{k_{1}} \cdots \lambda_{g}^{k_{g}}
$$


where $\psi_{i}=c_{1}\left(\mathbb{L}_{i}\right)$ is the first Chern class of $\mathbb{L}_{i}$, and $\lambda_{j}=c_{j}(\mathbb{E})$ is the $j$-th Chern class of $\mathbb{E}$. The dimension of $\overline{\mathcal{M}}_{g, n}$ is $3 g-3+n$, hence (4) is equal to zero unless $\sum_{i=1}^{n} j_{i}+\sum_{i=1}^{g} i k_{i}=3 g-3+n$. Let

$$
\Lambda_{g}^{\vee}(u)=u^{g}-\lambda_{1} u^{g-1}+\cdots+(-1)^{g} \lambda_{g}=\sum_{i=0}^{g}(-1)^{i} \lambda_{i} u^{g-i}
$$

be the Chern polynomial of the dual bundle $\mathbb{E}^{\vee}$ of $\mathbb{E}$. For any partition $\mu: \mu_{1} \geq \mu_{2} \geq$ $\cdots \geq \mu_{l(u)}>0$, define

$$
\begin{aligned}
\mathcal{C}_{g, \mu}(\tau)= & -\frac{\sqrt{-1}^{|\mu|+l(\mu)}}{|\operatorname{Aut}(\mu)|}[\tau(\tau+1)]^{l(\mu)-1} \prod_{i=1}^{l(\mu)} \frac{\prod_{a=1}^{\mu_{i}-1}\left(\mu_{i} \tau+a\right)}{\left(\mu_{i}-1\right) !} \\
& \cdot \int_{\overline{\mathcal{M}}_{g, n}} \frac{\Lambda_{g}^{\vee}(1) \Lambda_{g}^{\vee}(-\tau-1) \Lambda_{g}^{\vee}(\tau)}{\prod_{i=1}^{l(\mu)}\left(1-\mu_{i} \psi_{i}\right)} \\
\mathcal{C}_{\mu}(\lambda ; \tau)= & \sum_{g \geq 0} \lambda^{2 g-2+l(\mu)} \mathcal{C}_{g, \mu}(\tau),
\end{aligned}
$$

here $\tau$ is a formal variable. Note that

$$
\int_{\overline{\mathcal{M}}_{g, n}} \frac{\Lambda_{g}^{\vee}(1) \Lambda_{g}^{\vee}(-\tau-1) \Lambda_{g}^{\vee}(\tau)}{\prod_{i=1}^{l(\mu)}\left(1-\mu_{i} \psi_{i}\right)}=|\mu|^{l(\mu)-3}
$$

for $l(\mu) \geq 3$, and we use this expression to extend the definition to the case $l(\mu)<3$.

Introduce formal variables $p=\left(p_{1}, p_{2}, \cdots, p_{n}, \cdots\right)$, and define

$$
p_{\mu}=p_{\mu_{1}} \cdots p_{\mu_{l(\mu)}}
$$

for a partition $\mu$. Define generating functions

$$
\begin{aligned}
\mathcal{C}(\lambda ; \tau, p) & =\sum_{|\mu| \geq 1} \mathcal{C}_{\mu}(\lambda ; \tau) p_{\mu}, \\
\mathcal{C}(\lambda ; \tau, p)^{\bullet} & =e^{\mathcal{C}(\lambda ; \tau, p)} .
\end{aligned}
$$

In [6], Chiu-chu Melissa Liu, Kefeng Liu and Jian Zhou have proved the following formula which was conjectured by Mariño and Vafa in [10].

Theorem 2.1. (Mariño-Vafa Formula) For every partition $\mu$, we have

$$
\begin{aligned}
& \mathcal{C}(\lambda ; \tau, p)= \\
& \sum_{n \geq 1} \frac{(-1)^{n-1}}{n} \sum_{\mu}\left(\sum_{\cup_{i=1}^{n} \mu^{i}=\mu} \prod_{i=1}^{n} \sum_{\left|\nu^{i}\right|=\left|\mu^{i}\right|} \frac{\chi_{\nu^{i}}\left(C\left(\mu^{i}\right)\right)}{z_{\mu^{i}}} e^{\sqrt{-1}\left(\tau+\frac{1}{2}\right) \kappa_{\nu^{i}} \lambda / 2} V_{\nu^{i}}(\lambda)\right) p_{\mu}, \\
& \mathcal{C}(\lambda ; \tau, p)^{\bullet}=\sum_{|\mu| \geq 0}\left(\sum_{|\nu|=|\mu|} \frac{\chi_{\nu}(C(\mu))}{z_{\mu}} e^{\sqrt{-1}\left(\tau+\frac{1}{2}\right) \kappa_{\nu} \lambda / 2} V_{\nu}(\lambda)\right) p_{\mu},
\end{aligned}
$$

where

$$
V_{\nu}(\lambda)=\prod_{1 \leq a<b \leq l(\nu)} \frac{\sin \left[\left(\nu_{a}-\nu_{b}+b-a\right) \lambda / 2\right]}{\sin [(b-a) \lambda / 2]} \frac{1}{\prod_{i=1}^{l(\nu)} \prod_{v=1}^{\nu_{i}} 2 \sin [(v-i+l(\nu)) \lambda / 2]} .
$$


It is known that

$$
V_{\nu}(\lambda)=\frac{1}{2^{l(\nu)} \prod_{x \in \nu} \sin [h(x) \lambda / 2]} .
$$

2.3. Mumford's relations. Let $c_{t}(\mathbb{E})=\sum_{i=0}^{g} t^{i} \lambda_{i}$, then we have

$$
c_{-t}(\mathbb{E})=t^{g} \Lambda_{g}^{\vee}\left(\frac{1}{t}\right) .
$$

Mumford's relations [11] are given by

$$
c_{t}(\mathbb{E}) c_{-t}(\mathbb{E})=1
$$

equivalently

$$
\Lambda_{g}^{\vee}(t) \Lambda_{g}^{\vee}(-t)=(-1)^{g} t^{2 g}
$$

then

$$
\lambda_{k}^{2}=\sum_{i=1}^{k}(-1)^{i+1} 2 \lambda_{k-i} \lambda_{k+i}
$$

where $\lambda_{0}=1$ and $\lambda_{k}=0$ for $k>g$. Let $x_{1}, \cdots, x_{g}$ be the formal Chern roots of $\mathbb{E}$, the Chern character is defined by

$$
\operatorname{ch}(\mathbb{E})=\sum_{i=1}^{g} e^{x_{i}}=g+\sum_{n=1}^{+\infty} \sum_{i=1}^{g} \frac{x_{i}^{n}}{n !},
$$

we write

$$
\begin{aligned}
\operatorname{ch}_{0}(\mathbb{E}) & =g \\
\operatorname{ch}_{n}(\mathbb{E}) & =\frac{1}{n !} \sum_{i=1}^{g} x_{i}^{n}, \quad n=1,2, \cdots .
\end{aligned}
$$

From the above identities we have the relation between $\operatorname{ch}_{n}(\mathbb{E})$ and $\lambda_{n}$ :

$$
\begin{aligned}
n ! \operatorname{ch}_{n}(\mathbb{E}) & =\sum_{i+j=n}(-1)^{i-1} i \lambda_{i} \lambda_{j}, \quad n<2 g, \\
\operatorname{ch}_{n}(\mathbb{E}) & =0, \quad n \geq 2 g .
\end{aligned}
$$

It is easy to see that

$$
\begin{aligned}
& (2 g-1) ! \operatorname{ch}_{2 g-1}(\mathbb{E})=(-1)^{g-1} \lambda_{g-1} \lambda_{g}, \\
& (2 g-2) ! \operatorname{ch}_{2 g-2}(\mathbb{E})=(-1)^{g-1}\left((2 g-2) \lambda_{g-2} \lambda_{g}-(g-1) \lambda_{g-1}^{2}\right), \\
& (2 g-3) ! \operatorname{ch}_{2 g-3}(\mathbb{E})=(-1)^{g-1}\left(3 \lambda_{g-3} \lambda_{g}-\lambda_{g-1} \lambda_{g-2}\right) .
\end{aligned}
$$

2.4. Bernoulli numbers. The Bernoulli numbers $B_{m}$ are defined by the following series expansion:

$$
\frac{t}{e^{t}-1}=\sum_{m=0}^{+\infty} B_{m} \frac{t^{m}}{m !}
$$

the first few terms are given by

$$
B_{0}=1, \quad B_{1}=-\frac{1}{2}, \quad B_{2}=\frac{1}{6}, \quad B_{3}=0, \quad B_{4}=-\frac{1}{30}, \quad B_{5}=0, \quad B_{6}=\frac{1}{42} .
$$


Finally we recall two formulas which will be used later:

$$
\begin{aligned}
\frac{t / 2}{\sin (t / 2)} & =1+\sum_{g=1}^{+\infty} \frac{2^{2 g-1}-1}{2^{2 g-1}} \frac{\left|B_{2 g}\right|}{(2 g) !} t^{2 g}, \\
\sum_{i=1}^{d-1} i^{m} & =\sum_{k=0}^{m} \frac{\left(\begin{array}{c}
m+1 \\
k
\end{array}\right)}{m+1} B_{k} d^{m+1-k},
\end{aligned}
$$

where $m$ is a positive integer.

\section{Some New Results from Mariño-Vafa Formula}

In this section we derive some new results from the Mariño-Vafa formula, we will need two formulas in [7, 2.1 and 5.1].

Theorem 3.1. We have the following results:

$$
\begin{gathered}
\sum_{g \geq 0} \lambda^{2 g} \int_{\overline{\mathcal{M}}_{g, 1}} \frac{\left.\frac{d}{d \tau}\right|_{\tau=0}\left[\Lambda_{g}^{\vee}(1) \Lambda_{g}^{\vee}(\tau) \Lambda_{g}^{\vee}(-\tau-1)\right]}{1-d \psi_{1}} \\
=-\sum_{a=1}^{d-1} \frac{1}{a} \frac{d \lambda / 2}{d \sin (d \lambda / 2)}+\sum_{i+j=d, i, j \neq 0} \frac{\lambda^{2}}{8 \sin (i \lambda / 2) \sin (j \lambda / 2)}, \\
\left.\frac{d}{d \tau}\right|_{\tau=0}\left[\Lambda_{g}^{\vee}(1) \Lambda_{g}^{\vee}(\tau) \Lambda_{g}^{\vee}(-\tau-1)\right]=-\lambda_{g-1}-\lambda_{g} \sum_{k \geq 0} k !(-1)^{k-1} \operatorname{ch}_{k}(\mathbb{E}) .
\end{gathered}
$$

3.1. The coefficient of $\lambda^{2 g}$. Introduce the notation

$$
b_{g}=\left\{\begin{array}{cc}
1, & g=0 \\
\frac{2^{2 g-1}-1}{2^{2 g-1}} \frac{\left|B_{2 g}\right|}{(2 g) !}, & g>0
\end{array}\right.
$$

then the coefficient of $\lambda^{2 g}$ in $-\sum_{a=1}^{d-1} \frac{1}{a} \frac{d \lambda / 2}{d \sin (d \lambda / 2)}$ is

$$
\left(-\sum_{a=1}^{d-1} \frac{1}{a}\right) \cdot b_{g} d^{2 g-1}
$$

If $g_{1}, g_{2} \geq 0$ and $g_{1}+g_{2}=g$, define

$$
F_{g_{1}, g_{2}}(d)=\sum_{i+j=d, i, j \neq 0} i^{2 g_{1}-1} j^{2 g_{2}-1} .
$$

In [6] it is showed that if $g_{1}, g_{2} \geq 1$, then

$$
F_{g_{1}, g_{2}}(d)=\sum_{k=0}^{2 g_{2}-1} \sum_{l=0}^{2 g-2-k} \frac{(-1)^{2 g_{2}-1-k}}{2 g-1-k}\left(\begin{array}{c}
2 g_{2}-1 \\
k
\end{array}\right)\left(\begin{array}{c}
2 g-1-k \\
l
\end{array}\right) B_{l} d^{2 g-1-l},
$$


for the rest case we have

$$
\begin{aligned}
F_{0, g}(d) & =\sum_{i+j=d, i, j \neq 0} i^{-1} j^{2 g-1} \\
& =\sum_{i=1}^{d-1} i^{-1}(d-i)^{2 g-1} \\
& =\sum_{k=0}^{2 g-1}(-1)^{2 g-1-k}\left(\begin{array}{c}
2 g-1 \\
k
\end{array}\right) d^{k} \sum_{i=1}^{d-1} i^{2 g-2-k} \\
& =\sum_{k=0}^{2 g-3}(-1)^{2 g-1-k}\left(\begin{array}{c}
2 g-1 \\
k
\end{array}\right) d^{k} \sum_{l=0}^{2 g-k-2} \frac{(2 g-1-k}{2 g-1-k} B_{l} d^{2 g-1-k-l} \\
& -(2 g-1) d^{2 g-2}(d-1)+d^{2 g-1} \sum_{i=1}^{d-1} \frac{1}{i} \\
& =\sum_{k=0}^{2 g-2} \sum_{l=0}^{2 g-k-2}\left(\begin{array}{c}
2 g-1 \\
k
\end{array}\right)\left(\begin{array}{l}
2 g-1-k \\
l
\end{array}\right) \frac{(-1)^{2 g-1-k}}{2 g-1-k} B_{l} d^{2 g-1-l} \\
& +(2 g-1) d^{2 g-2}+d^{2 g-1} \sum_{i=1}^{d-1} \frac{1}{i} .
\end{aligned}
$$

Note that $F_{0, g}(d)=F_{g, 0}(d)$ and

$$
\sum_{i+j=d, i, j \neq 0} \frac{\lambda^{2}}{8 \sin (i \lambda / 2) \sin (j \lambda / 2)}=\frac{1}{2} \sum_{g \geq 0} \lambda^{2 g}\left(\sum_{g_{1}+g_{2}=g} b_{g_{1}} b_{g_{2}} F_{g_{1}, g_{2}}(d)\right) .
$$

3.2. The Main Theorem. Let

$$
\sum_{g \geq 0} \lambda^{2 g} L H S=\sum_{g \geq 0} \lambda^{2 g} \int_{\overline{\mathcal{M}}_{g, 1}} \frac{\left.\frac{d}{d \tau}\right|_{\tau=0}\left[\Lambda_{g}^{\vee}(1) \Lambda_{g}^{\vee}(\tau) \Lambda_{g}^{\vee}(-\tau-1)\right]}{1-d \psi_{1}},
$$

and

$$
\sum_{g \geq 0} \lambda^{2 g} R H S=-\sum_{a=1}^{d-1} \frac{1}{a} \frac{d \lambda / 2}{d \sin (d \lambda / 2)}+\sum_{i+j=d, i, j \neq 0} \frac{\lambda^{2}}{8 \sin (i \lambda / 2) \sin (j \lambda / 2)},
$$

then we have

$$
\begin{aligned}
& L H S=-\int_{\overline{\mathcal{M}}_{g, 1}}\left(\lambda_{g-1} \psi_{1}^{2 g-1}\right) d^{2 g-1} \\
& -\sum_{k=0}^{2 g-2}\left[(2 g-2-k) !(-1)^{2 g-3-k} \int_{\overline{\mathcal{M}}_{g, 1}} \lambda_{g} \operatorname{ch}_{2 g-2-k}(\mathbb{E}) \psi_{1}^{k}\right] d^{k}, \\
& \text { (28) } R H S=-\sum_{a=1}^{d-1} \frac{b_{g}}{a} d^{2 g-1}+b_{g} F_{0, g}(d)+\frac{1}{2} \sum_{g_{1}+g_{2}=g, g_{1}, g_{2}>0} b_{g_{1}} b_{g_{2}} F_{g_{1}, g_{2}}(d) \text {. }
\end{aligned}
$$

Hence we can derive our main theorem: 
Theorem 3.2. If $1 \leq m \leq 2 g-3$ and $g \geq 2$, then

$$
\begin{gathered}
-(2 g-2-m) !(-1)^{2 g-3-m} \int_{\overline{\mathcal{M}}_{g, 1}} \lambda_{g} \operatorname{ch}_{2 g-2-m}(\mathbb{E}) \psi_{1}^{m} \\
=b_{g} \sum_{k=0}^{m-1} \frac{(-1)^{2 g-1-k}}{2 g-1-k}\left(\begin{array}{c}
2 g-1 \\
k
\end{array}\right)\left(\begin{array}{c}
2 g-1-k \\
2 g-1-m
\end{array}\right) B_{2 g-1-m}+ \\
\frac{1}{2} \sum_{g_{1}+g_{2}=g, g_{1}, g_{2}>0} b_{g_{1}} b_{g_{2}} \sum_{k=0}^{\min \left(2 g_{2}-1, m-1\right)} \frac{(-1)^{2 g_{2}-1-k}}{2 g-1-k}\left(\begin{array}{c}
2 g_{2}-1 \\
k
\end{array}\right)\left(\begin{array}{c}
2 g-1-k \\
2 g-1-m
\end{array}\right) B_{2 g-1-m} .
\end{gathered}
$$

Remark 3.3. Liu-Liu-Zhou [7] have only considered the cases $m=2 g-1$ and $m=1$.

3.3. The case of $m=2 g-3$. If $m=2 g-3$, we find that $1 ! \operatorname{ch}_{1}(\mathbb{E})=\lambda_{1}$, then

$$
\begin{aligned}
& \text { LHS }=-\int_{\overline{\mathcal{M}}_{g, 1}} \lambda_{g} \lambda_{1} \psi_{1}^{2 g-3}, \\
& R H S=b_{g} \sum_{k=0}^{2 g-4} \frac{(-1)^{2 g-1-k}}{2 g-1-k}\left(\begin{array}{c}
2 g-1 \\
k
\end{array}\right)\left(\begin{array}{c}
2 g-1-k \\
2
\end{array}\right) B_{2} \\
& +\frac{1}{2} \sum_{g_{1}+g_{2}=g, g_{1}, g_{2}>0} b_{g_{1}} b_{g_{2}} \sum_{k=0}^{\min \left(2 g_{2}-1,2 g-4\right)} \frac{(-1)^{2 g_{2}-1-k}}{2 g-1-k}\left(\begin{array}{c}
2 g_{2}-1 \\
k
\end{array}\right)\left(\begin{array}{c}
2 g-1-k \\
2
\end{array}\right) B_{2} .
\end{aligned}
$$

From the above formula we obtain a new result of the Hodge integral.

Theorem 3.4. If $g \geq 2$, then

$$
\int_{\overline{\mathcal{M}}_{g, 1}} \lambda_{1} \lambda_{g} \psi_{1}^{2 g-3}=\frac{1}{12}\left[g(2 g-3) b_{g}+b_{1} b_{g-1}\right] .
$$

Proof. Note that

$$
\begin{gathered}
\int_{\overline{\mathcal{M}}_{g, 1}} \lambda_{1} \lambda_{g} \psi_{1}^{2 g-3}=-b_{g} B_{2} \sum_{k=0}^{2 g-4} \frac{(-1)^{2 g-1-k}}{2 g-1-k}\left(\begin{array}{c}
2 g-1 \\
k
\end{array}\right)\left(\begin{array}{c}
2 g-1-k \\
2
\end{array}\right) \\
-\frac{B_{2}}{2} \sum_{g_{1}+g_{2}=g, g_{1}, g_{2}>0} b_{g_{1}} b_{g_{2}} \sum_{k=0}^{\min \left(2 g_{2}-1,2 g-4\right)} \frac{(-1)^{2 g_{2}-1-k}}{2 g-1-k}\left(\begin{array}{c}
2 g_{2}-1 \\
k
\end{array}\right)\left(\begin{array}{c}
2 g-1-k \\
2
\end{array}\right),
\end{gathered}
$$

let us write

$$
\begin{aligned}
A_{1} & =\sum_{k=0}^{2 g-4} \frac{(-1)^{2 g-1-k}}{2 g-1-k}\left(\begin{array}{c}
2 g-1 \\
k
\end{array}\right)\left(\begin{array}{c}
2 g-1-k \\
2
\end{array}\right), \\
f_{1}(x) & =\sum_{k=0}^{2 g-3}(-1)^{2 g-1-k}\left(\begin{array}{c}
2 g-1 \\
k
\end{array}\right)(2 g-2-k) x^{2 g-3-k}, \\
g_{1}(x) & =\sum_{k=0}^{2 g-2}(-1)^{2 g-1-k}\left(\begin{array}{c}
2 g-1 \\
k
\end{array}\right) x^{2 g-2-k},
\end{aligned}
$$


then

$$
A_{1}=\frac{1}{2} \sum_{k=0}^{2 g-4}(-1)^{2 g-1-k}\left(\begin{array}{c}
2 g-1 \\
k
\end{array}\right)(2 g-2-k), x g_{1}(x)=(1-x)^{2 g-1}-1, f_{1}(x)=g_{1}^{\prime}(x) .
$$

Hence

$$
f_{1}(x)=\frac{(2 g-1) x(1-x)^{2 g-2}-(1-x)^{2 g-1}+1}{x^{2}}, \quad f_{1}(1)=1,
$$

and we obtain

$$
A_{1}=\frac{1}{2}\left[f_{1}(1)-\left(\begin{array}{c}
2 g-1 \\
2 g-3
\end{array}\right)\right]=-\frac{1}{2}\left[\left(\begin{array}{c}
2 g-1 \\
2 g-3
\end{array}\right)-1\right] .
$$

Similarly, we write

$$
A_{2}=\sum_{k=0}^{\min \left(2 g_{2}-1,2 g-4\right)} \frac{(-1)^{2 g_{2}-1-k}}{2 g-1-k}\left(\begin{array}{c}
2 g_{2}-1 \\
k
\end{array}\right)\left(\begin{array}{c}
2 g-1-k \\
2
\end{array}\right),
$$

then

$$
A_{2}=\left\{\begin{array}{cc}
\frac{1}{2} \sum_{k=0}^{2 g_{2}-1}(-1)^{2 g_{2}-1-k}(2 g-2-k)\left(\begin{array}{c}
2 g_{2}-1 \\
k
\end{array}\right), & g_{2} \leq g-2, \\
\frac{1}{2} \sum_{k=0}^{2 g-4}(-1)^{2 g-1-k}(2 g-2-k)\left(\begin{array}{c}
2 g-3 \\
k
\end{array}\right), & g_{2}=g-1 .
\end{array}\right.
$$

Case 1: $g_{2} \geq g-2$. Let

$$
\begin{aligned}
& f_{2}(x)=\sum_{k=0}^{2 g_{2}-1}(-1)^{2 g_{2}-1-k}(2 g-2-k)\left(\begin{array}{c}
2 g_{2}-1 \\
k
\end{array}\right) x^{2 g-3-k}, \\
& g_{2}(x)=\sum_{k=0}^{2 g_{2}-1}(-1)^{2 g_{2}-1-k}\left(\begin{array}{c}
2 g_{2}-1 \\
k
\end{array}\right) x^{2 g-2-k} .
\end{aligned}
$$

Since $g \geq g_{2}+2$, then $2 g-3-\left(2 g_{2}-1\right) \geq 2>0$ and $g_{2}^{\prime}(x)=f_{2}(x)$. On the other hand

$$
g_{2}(x)=(1-x)^{2 g_{2}-1} x^{2 g_{1}-1},
$$

hence

$$
g_{2}^{\prime}(x)=-\left(2 g_{2}-1\right)(1-x)^{2 g_{2}-2} x^{2 g_{1}-1}+\left(2 g_{1}-1\right)(1-x)^{2 g_{2}-1} x^{2 g_{1}-2}
$$

and

$$
f_{2}(1)=\left\{\begin{array}{c}
-1, \quad g_{2}=1 \\
0, \quad 1<g_{2} \leq g-2
\end{array}\right.
$$

Case 2: $g_{2}=g-1$. let

$$
\begin{aligned}
& f_{3}(x)=\sum_{k=0}^{2 g-4}(-1)^{2 g-1-k}(2 g-2-k)\left(\begin{array}{c}
2 g-3 \\
k
\end{array}\right) x^{2 g-3-k}, \\
& g_{3}(x)=\sum_{k=0}^{2 g-4}(-1)^{2 g-1-k}\left(\begin{array}{c}
2 g-3 \\
k
\end{array}\right) x^{2 g-2-k} .
\end{aligned}
$$

Since $2 g-3-(2 g-4)=1>0$,

$$
\frac{g_{3}(x)}{x}=\sum_{k=0}^{2 g-4}(-1)^{2 g-3-k}\left(\begin{array}{c}
2 g-3 \\
k
\end{array}\right) x^{2 g-3-k}=(1-x)^{2 g-3}-1
$$


and

$$
g_{3}^{\prime}(x)=(1-x)^{2 g-3}-1-(2 g-3)(1-x)^{2 g-4} x,
$$

therefore we have

$$
f_{3}(1)= \begin{cases}-2, & g=2, \\ -1, & g>2 .\end{cases}
$$

From the values of $f_{1}(1), f_{2}(1), f_{3}(1)$, we obtain

$$
\begin{aligned}
& \int_{\overline{\mathcal{M}}_{g, 1}} \lambda_{1} \lambda_{g} \psi_{1}^{2 g-3} \\
= & -b_{g} B_{2} A_{1}-\frac{B_{2}}{2}\left[\frac{1}{2} \sum_{g_{1}+g_{2}=g, 1 \leq g_{2} \leq g-2} b_{g_{1}} b_{g_{2}} f_{2}(1)+\frac{1}{2} b_{1} b_{g-1} f_{3}(1)\right] \\
= & \frac{B_{2}}{2}\left[-b_{g} A_{1}+b_{1} b_{g-1}\right] \\
= & \frac{1}{12}\left[g(2 g-3) b_{g}+b_{1} b_{g-1}\right]
\end{aligned}
$$

Since $B_{n}=0$ for $n$ odd and $n>1$, we also have the following vanishing result.

Theorem 3.5. If $g \geq 2$, then for any $1 \leq t \leq g-1$, we have

$$
\int_{\overline{\mathcal{M}}_{g, 1}} \lambda_{g} \operatorname{ch}_{2 t}(\mathbb{E}) \psi_{1}^{2(g-1-t)}=0 .
$$

\section{Another Simple Proof of $\lambda_{q}$ Conjecture}

Let $|\mu|=d, l(\mu)=n$, denote by $\left[\mathcal{C}_{g, \mu}(\tau)\right]_{k}$ the coefficient of $\tau^{k}$ in the polynomial $\mathcal{C}_{g, \mu}(\tau)$, and let

$$
\begin{aligned}
J_{g, \mu}^{0}(\tau) & =\sqrt{-1}^{|\mu|-l(\mu)} \mathcal{C}_{g, \mu}(\tau) \\
J_{g, \mu}^{1}(\tau) & =\sqrt{-1}^{|\mu|-l(\mu)-1}\left(\sum_{\nu \in J(\mu)} I_{1}(\nu) \mathcal{C}_{g, \nu}(\tau)+\sum_{\nu \in C(\mu)} I_{2}(\nu) \mathcal{C}_{g-1, \nu}(\tau)\right. \\
& \left.+\sum_{g_{1}+g_{2}=g, \nu^{1} \cup \nu^{2} \in C(\mu)} I_{3}\left(\nu^{1}, \nu^{2}\right) \mathcal{C}_{g_{1}, \nu^{1}}(\tau) \mathcal{C}_{g_{2}, \nu^{2}}(\tau)\right)
\end{aligned}
$$

The set $J(\mu)$ consists of partitions of $d$ of the form

$$
\nu=\left(\mu_{1}, \cdots, \widehat{\mu}_{i}, \cdots, \mu_{l(\mu)}, \mu_{i}+\mu_{j}\right)
$$

and the set $C(\mu)$ consists of partitions of $d$ of the form

$$
\nu=\left(\mu_{1}, \cdots, \widehat{\mu}_{i}, \cdots, \mu_{l(\mu)}, j, k\right)
$$

where $j+k=\mu_{i}$. The definitions of $I_{1}, I_{2}$ and $I_{3}$ can be found in [5]. Liu-Liu-Zhou [6] have proved the following differential equation:

$$
\frac{d}{d \tau} J_{g, \mu}^{0}(\tau)=-J_{g, \mu}^{1}(\tau)
$$


It is straightforward to check that

$$
\begin{aligned}
{\left[\mathcal{C}_{g, \mu}(\tau)\right]_{n-1} } & =-\frac{\sqrt{-1}^{d+n}}{|\operatorname{Aut}(\mu)|} \int_{\overline{\mathcal{M}}_{g, n}} \frac{\lambda_{g}}{\prod_{i=1}^{n}\left(1-\mu_{i} \psi_{i}\right)}, \\
{\left[\sum_{\nu \in J(\mu)} I_{1}(\nu) \mathcal{C}_{g, \nu}(\tau)\right]_{n-2} } & =-\frac{\sqrt{-1}^{d+n-1}}{|\operatorname{Aut}(\nu)|} \int_{\overline{\mathcal{M}}_{g, n-1}} \frac{\lambda_{g}}{\prod_{i=1}^{n-1}\left(1-\mu_{i} \psi_{i}\right)}, \\
\sum_{\left.\sum_{g_{1}+g_{2}=g, \nu^{1} \cup \nu^{2} \in C(\mu)} I_{3}\left(\nu^{1}, \nu^{2}\right) \mathcal{C}_{g_{1}, \nu^{1}}(\tau) \mathcal{C}_{g_{2}, \nu^{2}}(\tau)\right]_{n-2}}=0, & 0,
\end{aligned}
$$

hence, from (31) we have the identity

$$
\frac{n-1}{|\operatorname{Aut}(\mu)|} \int_{\overline{\mathcal{M}}_{g, n}} \frac{\lambda_{g}}{\prod_{i=1}^{n}\left(1-\mu_{i} \psi_{i}\right)}=\sum_{\nu \in J(\mu)} \frac{I_{1}(\nu)}{|\operatorname{Aut}(\nu)|} \int_{\overline{\mathcal{M}}_{g, n-1}} \frac{\lambda_{g}}{\prod_{i=1}^{n-1}\left(1-\nu_{i} \psi_{i}\right)}
$$

Theorem 4.1. For any partition $\mu: \mu_{1} \geq \mu_{2} \geq \cdots \geq \mu_{n}>0$ of $d$ and $g>0$, then

$$
\int_{\overline{\mathcal{M}}_{g, n}} \frac{\lambda_{g}}{\prod_{i=1}^{n}\left(1-\mu_{i} \psi_{i}\right)}=d^{2 g+n-3} b_{g}
$$

Proof. Recall the definition of $I_{1}(\nu)$, where $\nu=\left(\mu_{1}, \cdots, \widehat{\mu_{i}}, \cdots, \widehat{\mu}_{j}, \cdots, \mu_{n}, \mu_{i}+\mu_{j}\right)$ :

$$
I_{1}(\nu)=\frac{\mu_{i}+\mu_{j}}{1+\delta_{\mu_{j}}^{\mu_{i}}} m_{\mu_{i}+\mu_{j}}(\nu)
$$

and it is easy to see that

$$
\frac{m_{\mu_{i}+\mu_{j}}(\nu)}{|\operatorname{Aut}(\nu)|}=\frac{m_{\mu_{i}}(\mu)\left(m_{\mu_{j}}(\mu)-\delta_{\mu_{j}}^{\mu_{i}}\right)}{|\operatorname{Aut}(\mu)|}
$$

Let

$$
\mu: \underbrace{\mu_{k_{1}}=\cdots=\mu_{k_{1}}}_{t_{1}}>\underbrace{\mu_{k_{2}}=\cdots=\mu_{k_{2}}}_{t_{2}}>\cdots>\underbrace{\mu_{k_{s}}=\cdots=\mu_{k_{s}}}_{t_{s}}>0
$$

where

$$
\sum_{i=1}^{s} t_{i}=n, \quad \sum_{i=1}^{s} t_{i} \mu_{k_{i}}=d
$$


then

$$
\begin{aligned}
& \sum_{\nu \in J(\mu)} \frac{I_{1}(\nu)}{|\operatorname{Aut}(\nu)|} \\
= & \frac{1}{|\operatorname{Aut}(\mu)|} \sum_{\nu \in J(\mu)} \frac{\mu_{i}+\mu_{j}}{1+\delta_{\mu_{j}}^{\mu_{i}}}\left[m_{\mu_{i}}(\mu)\left(m_{\mu_{j}}(\mu)-\delta_{\mu_{j}}^{\mu_{i}}\right)\right] \\
= & \frac{1}{|\operatorname{Aut}(\mu)|}\left[\frac{1}{2} \sum_{i=1}^{s} \sum_{j \neq i}\left(\mu_{k_{i}}+\mu_{k_{j}}\right) m_{\mu_{k_{i}}}(\mu) m_{\mu_{k_{j}}}(\mu)+\sum_{i=1}^{s} \mu_{k_{i}} m_{\mu_{k_{i}}}(\mu)\left(m_{\mu_{k_{i}}}(\mu)-1\right)\right] \\
= & \frac{1}{|\operatorname{Aut}(\mu)|}\left[\frac{1}{2} \sum_{i=1}^{s} \sum_{j \neq i}\left(\mu_{k_{i}}+\mu_{k_{j}}\right) t_{i} t_{j}+\sum_{i=1}^{s} \mu_{k_{i}} t_{i}\left(t_{i}-1\right)\right] \\
= & \frac{1}{|\operatorname{Aut}(\mu)|}\left[\sum_{i=1}^{s} \sum_{j \neq i} \mu_{k_{j}} t_{j} t_{i}+\sum_{i=1}^{s} \mu_{k_{i}} t_{i}^{2}-d\right] \\
= & \frac{1}{|\operatorname{Aut}(\mu)|}\left[\sum_{i=1}^{s} t_{i}\left(d-\mu_{k_{i}} t_{i}\right)+\sum_{i=1}^{s} \mu_{k_{i}} t_{i}^{2}-d\right] \\
= & \frac{(n-1) d}{|\operatorname{Aut}(\mu)|} .
\end{aligned}
$$

By the induction of $n$ and the initial value of the Mariño-Vafa formula

$$
\int_{\overline{\mathcal{M}}_{g, 1}} \frac{\lambda_{g}}{1-\mu_{1} \psi_{1}}=d^{2 g-2} b_{g}
$$

we have

$$
\int_{\overline{\mathcal{M}}_{g, n}} \frac{\lambda_{g}}{\prod_{i=1}^{n}\left(1-\mu_{i} \psi_{i}\right)}=d \cdot d^{2 g+n-1-3} b_{g}=d^{2 g+n-3} b_{g}
$$

Corollary 4.2. The following $\lambda_{g}$ conjecture is true:

$$
\int_{\overline{\mathcal{M}}_{g, n}} \lambda_{g} \prod_{l=1}^{n} \psi_{l}^{k_{l}}=\left(\begin{array}{c}
2 g+n-3 \\
k_{1}, \cdots, k_{n}
\end{array}\right) b_{g},
$$

where $g>0$.

\section{A recursion Formula of the $\lambda_{g-1}$ Integral}

E.Getzler, A.Okounkov and R.Pandharipande have derived explicit formula for the multipoint series of $\mathbb{C P}^{1}$ in degree 0 from the Toda hierarchy [2], then they obtained certain formulas for the Hodge integrals $\int_{\overline{\mathcal{M}}_{g, n}} \lambda_{g-1} \psi_{1}^{k_{1}} \cdots \psi_{n}^{k_{n}}$. In this section we give an effective recursion formula of the $\lambda_{g-1}$ integrals using Mariño-Vafa formula. It is straightforward to check the following lemma. 
Lemma 5.1. We have the following identities

$$
\begin{aligned}
{\left[\prod_{i=1}^{n} \frac{\prod_{a=1}^{\mu_{i}-1}\left(\mu_{i} \tau+a\right)}{\left(\mu_{i}-1\right) !}\right]_{0} } & =1, \\
{\left[\prod_{i=1}^{n} \frac{\prod_{a=1}^{\mu_{i}-1}\left(\mu_{i} \tau+a\right)}{\left(\mu_{i}-1\right) !}\right]_{1} } & =\sum_{i=1}^{n} \sum_{a=1}^{\mu_{i}-1} \frac{\mu_{i}}{a}, \\
{\left[\Lambda_{g}^{\vee}(1) \Lambda_{g}^{\vee}(-\tau-1) \Lambda_{g}^{\vee}(\tau)\right]_{0} } & =\lambda_{g}, \\
{\left[\Lambda_{g}^{\vee}(1) \Lambda_{g}^{\vee}(-\tau-1) \Lambda_{g}^{\vee}(\tau)\right]_{0} } & =-\lambda_{g-1}-\sum_{k \geq 0} k !(-1)^{k-1} \operatorname{ch}_{k}(\mathbb{E}) \lambda_{g},
\end{aligned}
$$

and

$$
\begin{aligned}
{\left[\mathcal{C}_{g, \mu}(\tau)\right]_{n-1} } & =-\frac{\sqrt{-1}^{d+n}}{|\operatorname{Aut}(\mu)|} \int_{\overline{\mathcal{M}}_{g, n}} \frac{\lambda_{g}}{\prod_{i=1}^{n}\left(1-\mu_{i} \psi_{i}\right)} \\
{\left[\mathcal{C}_{g, \mu}(\tau)\right]_{n} } & =-\frac{\sqrt{-1}^{d+n}}{|\operatorname{Aut}(\mu)|}\left[n-1+\sum_{i=1}^{n} \sum_{a=1}^{\mu_{i}-1} \frac{\mu_{i}}{a}\right] \int_{\overline{\mathcal{M}}_{g, n}} \frac{\lambda_{g}}{\prod_{i=1}^{n}\left(1-\mu_{i} \psi_{i}\right)} \\
& +\frac{\sqrt{-1}^{d+n}}{|\operatorname{Aut}(\mu)|} \int_{\overline{\mathcal{M}}_{g, n}} \frac{\lambda_{g-1}+\sum_{k \geq 0} k !(-1)^{k-1} \operatorname{ch}_{k}(\mathbb{E}) \lambda_{g}}{\prod_{i=1}^{n}\left(1-\mu_{i} \psi_{i}\right)}
\end{aligned}
$$

Now, we can state our main theorem in this section using equation (31).

Theorem 5.2. For any partition $\mu$ with $l(\mu)=n$, we have the following recursion formula

$$
\begin{aligned}
& \frac{n}{|\operatorname{Aut}(\mu)|}\left[n-1+\sum_{i=1}^{n} \sum_{a=1}^{\mu_{i}-1} \frac{\mu_{i}}{a}\right] \int_{\overline{\mathcal{M}}_{g, n}} \frac{\lambda_{g}}{\prod_{i=1}^{n}\left(1-\mu_{i} \psi_{i}\right)} \\
- & \frac{n}{|\operatorname{Aut}(\mu)|} \int_{\overline{\mathcal{M}}_{g, n}} \frac{\lambda_{g-1}+\sum_{k \geq 0} k !(-1)^{k-1} \operatorname{ch}_{k}(\mathbb{E}) \lambda_{g}}{\prod_{i=1}^{n}\left(1-\mu_{i} \psi_{i}\right)} \\
= & \sum_{\nu \in J(\mu)} \frac{I_{1}(\nu)}{|\operatorname{Aut}(\nu)|}\left[n-2+\sum_{i=1}^{n-1} \sum_{a=1}^{\nu_{i}-1} \frac{\nu_{i}}{a}\right] \int_{\overline{\mathcal{M}}_{g, n-1}} \frac{\lambda_{g}}{\prod_{i=1}^{n-1}\left(1-\nu_{i} \psi_{i}\right)} \\
- & \sum_{\nu \in J(\mu)} \frac{I_{1}(\nu)}{|\operatorname{Aut}(\nu)|} \int_{\overline{\mathcal{M}}_{g, n-1}} \frac{\lambda_{g-1}+\sum_{k \geq 0} k !(-1)^{k-1} \operatorname{ch}_{k}(\mathbb{E}) \lambda_{g}}{\prod_{i=1}^{n-1}\left(1-\nu_{i} \psi_{i}\right)} \\
+ & \sum_{g_{1}+g_{2}=g, g_{1}, g_{2} \geq 0} \sum_{\nu^{1} \cup \nu^{2} \in C(\mu)} \frac{I_{3}\left(\nu^{1}, \nu^{2}\right)}{\lambda_{g_{1}}} \\
& \int_{\overline{\mathcal{M}}_{g_{1}, n_{1}}} \frac{\operatorname{Aut}_{\left.\nu^{1}\right)|| \operatorname{Aut}\left(\nu^{2}\right) \mid}^{\prod_{i=1}^{n_{1}}\left(1-\nu_{i}^{1} \psi_{i}\right)}}{\int_{\overline{\mathcal{M}}_{g_{2}, n_{2}}} \frac{\lambda_{g_{2}}}{\prod_{i=1}^{n_{2}}\left(1-\nu_{i}^{2} \psi_{i}\right)} .}
\end{aligned}
$$

5.1. The $\lambda_{g}$-Integral. In this subsection, we re-derive the $\lambda_{g}$-integral from theorem 5.2.. Let $\mu_{i}=N x_{i}$ for some $N \in \mathbb{N}$ and $x_{i} \in \mathbb{R}$, from Kim-Liu[4]'s method and 
consider the coefficients of $\ln N N^{2 g+n-2}$ in theorem 5.2., then

$$
\begin{aligned}
& n\left(x_{1}+\cdots+x_{n}\right) \prod_{l=1}^{n} x_{l}^{k_{l}} \int_{\overline{\mathcal{M}}_{g, n}} \lambda_{g} \prod_{l=1}^{n} \psi_{l}^{k_{l}} \\
= & \frac{1}{2} \sum_{i=1}^{n} \sum_{j \neq i}\left(x_{i}+x_{j}\right)^{k_{i}+k_{j}}\left(x_{1}+\cdots+x_{n}\right) \prod_{l \neq i, j} x_{l}^{k_{l}} \int_{\overline{\mathcal{M}}_{g, n-1}} \lambda_{g} \psi^{k_{i}+k_{j}-1} \prod_{l \neq i, j} \psi_{l}^{k_{l}} \\
+ & \left(x_{1}+\cdots+x_{n}\right) \prod_{l=1}^{n} x_{l}^{k_{l}} \int_{\overline{\mathcal{M}}_{g, n}} \lambda_{g} \prod_{l=1}^{n} \psi_{l}^{k_{l}}
\end{aligned}
$$

i.e.

$$
\begin{aligned}
& (n-1) \prod_{l=1}^{n} x_{l}^{k_{l}} \int_{\overline{\mathcal{M}}_{g, n}} \lambda_{g} \prod_{l=1}^{n} \psi_{l}^{k_{l}} \\
= & \frac{1}{2} \sum_{i=1}^{n} \sum_{j \neq i}\left(x_{i}+x_{j}\right)^{k_{i}+k_{j}} \prod_{l \neq i, j} x_{l}^{k_{l}} \int_{\overline{\mathcal{M}}_{g, n-1}} \lambda_{g} \psi^{k_{i}+k_{j}-1} \prod_{l \neq i, j} \psi_{l}^{k_{l}} .
\end{aligned}
$$

After introducing the formal variables $s_{l} \in \mathbb{R}^{+}$and applying the Laplace transformation

$$
\int_{0}^{+\infty} x^{k} e^{-x / 2 s} d x=k !(2 s)^{k+1}, \quad s>0,
$$

we select the coefficient of $\prod_{l=1}^{n}\left(2 s_{l}\right)^{k_{l}+1}$ from the transformation of (35), then we derive

$$
(n-1) \int_{\overline{\mathcal{M}}_{g, n}} \lambda_{g} \prod_{l=1}^{n} \psi_{l}^{k_{l}}=\frac{1}{2} \sum_{i=1}^{n} \sum_{j \neq i} \frac{\left(k_{i}+k_{j}\right) !}{k_{i} ! k_{j} !} \int_{\overline{\mathcal{M}}_{g, n-1}} \lambda_{g} \psi^{k_{i}+k_{j}-1} \prod_{l \neq i, j} \psi_{l}^{k_{l}} .
$$

By the induction of $n$, we obtain the $\lambda_{g}$ conjecture

$$
\int_{\overline{\mathcal{M}}_{g, n}} \lambda_{g} \prod_{l=1}^{n} \psi_{l}^{k_{l}}=\left(\begin{array}{c}
2 g+n-3 \\
k_{1}, \cdots, k_{n}
\end{array}\right) b_{g}
$$

in fact, in (36) we have

$$
\begin{aligned}
R H S & =\frac{1}{2} \sum_{i=1}^{n} \sum_{j \neq i} \frac{\left(k_{i}+k_{j}\right) !}{k_{i} ! k_{j} !} \frac{(2 g+n-4) !}{\prod_{l \neq i, j} k_{l} !\left(k_{i}+k_{j}-1\right) !} b_{g} \\
& =\frac{1}{2} \sum_{i=1}^{n} \sum_{j \neq i} \frac{k_{i}+k_{j}}{2 g+n-3}\left(\begin{array}{c}
2 g+n-3 \\
k_{1}, \cdots, k_{n}
\end{array}\right) b_{g},
\end{aligned}
$$

note that $k_{1}+\cdots+k_{n}=2 g+n-3$, therefore

$$
\begin{aligned}
\frac{1}{2} \sum_{i=1}^{n} \sum_{j \neq i}\left(k_{i}+k_{j}\right) & =\frac{1}{2} \sum_{i=1}^{n}\left[(n-1) k_{i}+\left(2 g+n-3-k_{i}\right)\right] \\
& =\frac{1}{2}[(n-2)(2 g+n-3)+(2 g+n-3) n] \\
& =\frac{1}{2}[(2 n-2)(2 g+n-3)] \\
& =(n-1)(2 g+n-3)
\end{aligned}
$$


5.2. The Recursion Formula of $\lambda_{g-1}$-integral. We have found the singular part $\sum_{i=1}^{n} \sum_{a=1}^{\mu_{i}-1} \frac{\mu_{i}}{a}$ in theorem 5.2., using the following theorem, we can eliminate this part and derive the recursion formula of $\lambda_{g-1}$-integral. The notation $[F]_{\text {sing }}$ means the singular part of $F$. First, in theorem 5.2., we have

$$
\begin{aligned}
{\left[\frac{L H S}{d^{2 g+n-4} b_{g}}\right]_{s i n g} } & =n \sum_{i=1}^{n} \sum_{a=1}^{\mu_{i}-1} \frac{\mu_{i}}{a} d, \\
{\left[\frac{R H S}{d^{2 g+n-4} b_{g}}\right]_{s i n g} } & =\frac{1}{2} \sum_{i=1}^{n} \sum_{j \neq i}\left(\mu_{i}+\mu_{j}\right)\left[\sum_{l \neq i, j} \sum_{a=1}^{\mu_{l}-1} \frac{\mu_{l}}{a}+\left(\mu_{i}+\mu_{j}\right) \sum_{a=1}^{\mu_{i}+\mu_{j}-1} \frac{1}{a}\right] \\
& +\sum_{i=1}^{n} \sum_{a=1}^{\mu_{i}-1} \frac{\mu_{i}}{a} d-\sum_{i=1}^{n} \sum_{j \neq i}^{\mu_{i}+\mu_{j}-1} \sum_{a=\mu_{j}+1}^{\mu_{j}\left(\mu_{i}+\mu_{j}\right)}
\end{aligned}
$$

Theorem 5.3. Under the above notation, we have

$$
\left[\frac{R H S}{d^{2 g+n-4} b_{g}}\right]_{\text {sing }}=\left[\frac{L H S}{d^{2 g+n-4} b_{g}}\right]_{\text {sing }}+2(n-1) d \text {. }
$$

Proof. Since

$$
\begin{aligned}
& \sum_{i=1}^{n} \sum_{j \neq i} \sum_{a=\mu_{j}+1}^{\mu_{i}+\mu_{j}-1} \frac{\mu_{j}\left(\mu_{i}+\mu_{j}\right)}{a} \\
= & \sum_{i=1}^{n} \sum_{j \neq i} \sum_{a=\mu_{j}+1}^{\mu_{i}+\mu_{j}-1} \frac{\left(\mu_{i}+\mu_{j}\right)^{2}}{a}-\sum_{i=1}^{n} \sum_{j \neq i} \sum_{a=\mu_{j}+1}^{\mu_{i}+\mu_{j}-1} \frac{\mu_{i}\left(\mu_{i}+\mu_{j}\right)}{a} \\
= & \sum_{i=1}^{n} \sum_{j \neq i} \sum_{a=1}^{\mu_{i}+\mu_{j}-1} \frac{\left(\mu_{i}+\mu_{j}\right)^{2}}{a}-\sum_{i=1}^{n} \sum_{j \neq i} \sum_{a=1}^{\mu_{j}} \frac{\left(\mu_{i}+\mu_{j}\right)^{2}}{a} \\
- & \sum_{i=1}^{n} \mu_{i} \sum_{j \neq i}^{\mu_{i}+\mu_{j}-1} \sum_{a=1}^{n} \frac{\mu_{i}+\mu_{j}}{a}+\sum_{i=1}^{n} \mu_{i} \sum_{j \neq i} \sum_{a=1}^{\mu_{j}} \frac{\left(\mu_{i}+\mu_{j}\right)}{a} \\
= & \sum_{i=1}^{n} \sum_{j \neq i}^{\mu_{i}+\mu_{j}-1} \sum_{a=1}^{\mu_{j}-1} \frac{\left(\mu_{i}+\mu_{j}\right)^{2}}{a}-\sum_{i=1}^{n} \sum_{j \neq i} \sum_{a=1}^{n} \frac{\left(\mu_{i}+\mu_{j}\right)^{2}}{a} \\
+ & \sum_{i=1}^{n} \mu_{i} \sum_{j \neq i} \sum_{a=1}^{\mu_{j}-1} \frac{\left(\mu_{i}+\mu_{j}\right)}{a}-\sum_{i=1}^{n} \sum_{j \neq i}\left(\mu_{i}+\mu_{j}\right),
\end{aligned}
$$

where we use the identity

$$
\begin{aligned}
\sum_{i=1}^{n} \sum_{j \neq i} \sum_{a=1}^{\mu_{i}+\mu_{j}-1} \frac{\left(\mu_{i}+\mu_{j}\right) \mu_{i}}{a} & =\sum_{i=1}^{n} \sum_{j \neq i} \sum_{a=1}^{\mu_{i}+\mu_{j}-1} \frac{\left(\mu_{i}+\mu_{j}\right) \mu_{j}}{a} \\
& =\frac{1}{2} \sum_{i=1}^{n} \sum_{j \neq i} \sum_{a=1}^{\mu_{i}+\mu_{j}-1} \frac{\left(\mu_{i}+\mu_{j}\right)^{2}}{a}
\end{aligned}
$$


Note that $\sum_{i=1}^{n} \sum_{j \neq i}\left(\mu_{i}+\mu_{j}\right)=2(n-1) d$, hence

$$
\begin{aligned}
& {\left[\frac{R H S}{d^{2 g+n-4} b_{g}}\right]_{\text {sing }} } \\
= & \frac{1}{2} \sum_{i=1}^{n} \sum_{j \neq i}\left(\mu_{i}+\mu_{j}\right) \sum_{l \neq i, j} \sum_{a=1}^{\mu_{l}-1} \frac{\mu_{l}}{a}+\sum_{i=1}^{n} \sum_{a=1}^{\mu_{i}-1} \frac{\mu_{i}}{a} d \\
+ & \sum_{i=1}^{n} \sum_{j \neq i} \sum_{a=1}^{\mu_{j}-1} \frac{\left(\mu_{i}+\mu_{j}\right)^{2}}{a}-\sum_{i=1}^{n} \sum_{j \neq i} \mu_{i} \sum_{a=1}^{\mu_{j}-1} \frac{\left(\mu_{i}+\mu_{j}\right)}{a}+\sum_{i=1}^{n} \sum_{j \neq i}\left(\mu_{i}+\mu_{j}\right) \\
= & \left(\sum_{i=1}^{n} \sum_{a=1}^{\mu_{i}-1} \frac{\mu_{i}}{a}\right) d+\frac{1}{2} \sum_{i=1}^{n} \sum_{j \neq i}\left(\mu_{i}+\mu_{j}\right) \sum_{l \neq i, j} \sum_{a=1}^{\mu_{l}-1} \frac{\mu_{l}}{a} \\
+ & \sum_{i=1}^{n} \sum_{j \neq i} \sum_{a=1}^{\mu_{j}-1} \frac{\mu_{j}\left(\mu_{i}+\mu_{j}\right)}{a}+2(n-1) d,
\end{aligned}
$$

it is straightforward to check that

$$
\begin{aligned}
\frac{1}{2} \sum_{i=1}^{n} \sum_{j \neq i}\left(\mu_{i}+\mu_{j}\right) \sum_{l \neq i, j} \sum_{a=1}^{\mu_{l}-1} \frac{\mu_{l}}{a} & =(n-2) \sum_{i=1}^{n} \mu_{i} \sum_{j \neq i} \sum_{a=1}^{\mu_{j}-1} \frac{\mu_{j}}{a} \\
\sum_{i=1}^{n} \sum_{j \neq i} \sum_{a=1}^{\mu_{j}-1} \frac{\mu_{j}\left(\mu_{i}+\mu_{j}\right)}{a} & =\sum_{i=1}^{n} \mu_{i} \sum_{j \neq i} \sum_{a=1}^{\mu_{j}-1} \frac{\mu_{j}}{a}+\sum_{i=1}^{n} \sum_{j \neq i} \mu_{j} \sum_{a=1}^{\mu_{j}-1} \frac{\mu_{j}}{a}, \\
\sum_{i=1}^{n} \sum_{j \neq i} \mu_{j} \sum_{a=1}^{\mu_{j}-1} \frac{\mu_{j}}{a} & =(n-1) \sum_{i=1}^{n} \mu_{i} \sum_{a=1}^{\mu_{i}-1} \frac{\mu_{i}}{a} .
\end{aligned}
$$

Finally, we obtain

$$
\begin{aligned}
& {\left[\frac{R H S}{d^{2 g+n-4} b_{g}}\right]_{\text {sing }} } \\
= & \left(\sum_{i=1}^{n} \sum_{a=1}^{\mu_{i}-1} \frac{\mu_{i}}{a}\right) d+(n-2) \sum_{i=1}^{n} \mu_{i} \sum_{j \neq i} \sum_{a=1}^{\mu_{j}-1} \frac{\mu_{j}}{a} \\
+ & \sum_{i=1}^{n} \mu_{i} \sum_{j \neq i} \sum_{a=1}^{\mu_{j}-1} \frac{\mu_{j}}{a}+(n-1) \sum_{i=1}^{n} \mu_{i} \sum_{a=1}^{\mu_{i}-1} \frac{\mu_{i}}{a}+2(n-1) d \\
= & \left(\sum_{i=1}^{n} \sum_{a=1}^{\mu_{i}-1} \frac{\mu_{i}}{a}\right) d+(n-1) \sum_{i=1}^{n} \mu_{i}\left(\sum_{j \neq i} \sum_{a=1}^{\mu_{i}-1} \frac{\mu_{j}}{a}+\sum_{a=1}^{\mu_{i}-1} \frac{\mu_{i}}{a}\right)+2(n-1) d \\
= & \left(\sum_{i=1}^{n} \sum_{a=1}^{\mu_{i}-1} \frac{\mu_{i}}{a}\right) d+(n-1)\left(\sum_{i=1}^{n} \sum_{a=1}^{\mu_{i}-1} \frac{\mu_{i}}{a}\right)^{d} d+2(n-1) d \\
= & {\left[\frac{L H S}{d^{2 g+n-4} b_{g}}\right]_{\operatorname{sing}}+2(n-1) d . }
\end{aligned}
$$


Let $\mathbb{R}^{k}\left[\mu_{1}, \cdots, \mu_{n}\right]$ be the space of all homogeneous polynomials with real coefficients in $\mu_{1}, \cdots, \mu_{n}$ of degree $k$, then it is the subring of $\mathbb{R}\left[\mu_{1}, \cdots, \mu_{n}\right]$. From the Theorem 5.3, we obtain the recursion formula of $\lambda_{g-1}$ Hodge integral.

Theorem 5.4. For any partition $\mu$ with $l(\mu)=n$ and $|\mu|=d$, we have the recursion formula

$$
\begin{aligned}
& \frac{n}{|\operatorname{Aut}(\mu)|} \int_{\overline{\mathcal{M}}_{g, n}} \frac{\lambda_{g-1}}{\prod_{i=1}^{n}\left(1-\mu_{i} \psi_{i}\right)} \\
= & \sum_{\nu \in J(\mu)} \frac{I_{1}(\nu)}{|\operatorname{Aut}(\nu)|} \int_{\overline{\mathcal{M}}_{g, n-1}} \frac{\lambda_{g-1}}{\prod_{i=1}^{n-1}\left(1-\nu_{i} \psi_{i}\right)} \\
-\quad & \sum_{g_{1}+g_{2}=g, \nu^{1} \cup \nu^{2} \in C(\mu)} \frac{I_{3}\left(\nu^{1}, \nu^{2}\right)}{\left|\operatorname{Aut}\left(\nu^{1}\right)\right|\left|\operatorname{Aut}\left(\nu^{2}\right)\right|} d_{1}^{2 g_{1}+n_{1}-3} d_{2}^{2 g_{2}+n_{2}-3} b_{g_{1}} b_{g_{2}} .
\end{aligned}
$$

under the ring $\mathbb{R}^{2 g-2+n}\left[\mu_{1}, \cdots, \mu_{n}\right]$, where $l\left(\nu^{i}\right)=n_{i}$ and $\left|\nu^{i}\right|=d_{i}$ for $i=1,2$.

Remark 5.5. When we consider the simplest case $n=1$, the above identity become the formula used in [6].

\section{Some Examples of The Main Theorem}

In this section we give some examples of theorem 3.2 .

6.1. The case of $g=3$. If $g=3$, then $1 \leq m \leq 3$. We consider three cases.

6.1.1. $m=1$. $L H S=-3 \int_{\overline{\mathcal{M}}_{3,1}} \lambda_{3} \operatorname{ch}_{3}(\mathbb{E}) \psi_{1}$, and $3 \operatorname{ch}_{3}(\mathbb{E})=\sum_{i+j=3}(-1)^{i-1} i \lambda_{i} \lambda_{j}=$ $3 \lambda_{3}-\lambda_{1} \lambda_{2}$, then we get

$$
\begin{aligned}
L H S & =\int_{\overline{\mathcal{M}}_{3,1}} \lambda_{3}\left(\lambda_{1} \lambda_{2}-3 \lambda_{3}\right) \psi_{1}=\int_{\overline{\mathcal{M}}_{3,1}} \lambda_{1} \lambda_{2} \lambda_{3} \psi_{1} \\
R H S & =b_{3} \frac{(-1)^{5}}{5}\left(\begin{array}{l}
5 \\
0
\end{array}\right)\left(\begin{array}{l}
5 \\
4
\end{array}\right) B_{4}+\frac{1}{2} \sum_{g_{1}+g_{2}=3, g_{1}, g_{2}>0} b_{g_{1}} b_{g_{2}} \frac{-1}{5}\left(\begin{array}{c}
2 g_{2}-1 \\
0
\end{array}\right)\left(\begin{array}{l}
5 \\
4
\end{array}\right) B_{4} \\
& =-B_{4}\left(b_{3}+b_{1} b_{2}\right) .
\end{aligned}
$$

Since $b_{1}=\frac{1}{24}, b_{2}=\frac{7}{5760}, b_{3}=\frac{31}{967680}$, we have

$$
\int_{\overline{\mathcal{M}}_{3,1}} \lambda_{1} \lambda_{2} \lambda_{3} \psi_{1}=\frac{1}{362880} .
$$

6.1.2. $m=2$. In this case we have $L H S=2 \int_{\overline{\mathcal{M}}_{3,1}} \lambda_{3} \operatorname{ch}_{2}(\mathbb{E}) \psi_{1}^{2}$, and $2 ! \operatorname{ch}_{2}(\mathbb{E})=2 \lambda_{2}-$ $\lambda_{1}^{2}, B_{3}=0$. Then we have

$$
\begin{aligned}
L H S & =\int_{\overline{\mathcal{M}}_{3,1}}\left(2 \lambda_{2} \lambda_{3}-\lambda_{3} \lambda_{1}^{2}\right) \psi_{1}^{2}, \\
R H S & =b_{3} \sum_{k=0}^{1} \frac{(-1)^{4-k}}{5-k}\left(\begin{array}{c}
5 \\
k
\end{array}\right)\left(\begin{array}{c}
5-k \\
3
\end{array}\right) B_{3} \\
& +\frac{1}{2} \sum_{g_{1}+g_{2}=3, g_{1}, g_{2}>0} b_{g_{1}} b_{g_{2}} \sum_{k=0}^{1} \frac{(-1)^{5-k}}{5-k}\left(\begin{array}{c}
2 g_{2}-1 \\
k
\end{array}\right)\left(\begin{array}{c}
5-k \\
3
\end{array}\right) B_{3} \\
& =0,
\end{aligned}
$$


hence

$$
\int_{\overline{\mathcal{M}}_{3,1}} \lambda_{3} \lambda_{1}^{2} \psi_{1}^{2}=2 \int_{\overline{\mathcal{M}}_{3,1}} \lambda_{2} \lambda_{3} \psi_{1}^{2} .
$$

Using the formula $\int_{\overline{\mathcal{M}}_{3,1}} \lambda_{2} \lambda_{3} \psi_{1}^{2}=\frac{1}{120960}$, we get

$$
\int_{\overline{\mathcal{M}}_{3,1}} \lambda_{3} \lambda_{1}^{2} \psi_{1}^{2}=\frac{1}{60480} .
$$

6.1.3. $m=3$. In this case $L H S=-\int_{\overline{\mathcal{M}}_{3,1}} \lambda_{3} \operatorname{ch}_{1}(\mathbb{E}) \psi_{1}^{3}$ and $\operatorname{ch}_{1}(\mathbb{E})=\lambda_{1}$, hence

$$
\begin{aligned}
\text { LHS } & =-\int_{\overline{\mathcal{M}}_{3,1}} \lambda_{1} \lambda_{3} \psi_{1}^{3} \\
\text { RHS } & =b_{3} \sum_{k=0}^{2} \frac{(-1)^{5-k}}{5-k}\left(\begin{array}{l}
5 \\
k
\end{array}\right)\left(\begin{array}{c}
5-k \\
2
\end{array}\right) B_{2} \\
& +\frac{1}{2} \sum_{g_{1}+g_{2}=3, g_{1}, g_{2}>0} b_{g_{1}} b_{g_{2}} \sum_{k=0}^{\min \left(2 g_{2}-1,2\right)} \frac{(-1)^{2 g_{2}-1-k}}{5-k}\left(\begin{array}{c}
2 g_{2}-1 \\
k
\end{array}\right)\left(\begin{array}{c}
5-k \\
2
\end{array}\right) B_{2} \\
& =-\frac{9}{2} b_{3} B_{2}-\frac{1}{2} b_{1} b_{2} B_{2} \\
& =-\frac{41}{1451520},
\end{aligned}
$$

SO

$$
\int_{\overline{\mathcal{M}}_{3,1}} \lambda_{1} \lambda_{3} \psi_{1}^{3}=\frac{41}{145120} .
$$

Remark 6.1. The values of (37) and (39) match with the results in [9], the identity (38) is a new result.

\section{Acknowledgements}

The author would like to thank his advisor, Professor Kefeng Liu, for his encouragement, helpful discussion on Hodge integrals and correction of grammar mistakes. I also thank Professor Edna Cheung who teach me a lot both in physics and in typing articles. Professor Chiu-Chu Liu commented on this note and pointed out some mistakes.

\section{References}

[1] C.Faber, R. Pandharipande. Hodge integrals and Gromov-Witten theory, Invent. Math. 139 (2000), 173-199.

[2] Hodge integrals, partition matrices, and the $\lambda_{g}$ conjecture, Ann. Math. (2) 157 (2003), 97-124. E. Getzler, A. Okounkov, R. Pandharipande. Multipoint series of Gromov-Witten invariants of $\mathbb{C P}^{1}$, preprint: math.AG/0207106.

[3] E. Getzler, A. Okounkov, R. Pandharipande. Multipoint series of Gromov-Witten invariants of $\mathbb{C P}^{1}$, preprint: math.AG/0207106.

[4] Y.-S. Kim, K. Liu. A simple proof of Witten conjecture through localization, preprint: math.AG/0508384.

[5] A.M. Li, G.Zhao, Q. Zheng. The number of ramifed coverings of a Riemann surface by Riemann surface, Comm.Math.Phys. 213 (2000), 685-696. 
[6] C.-C. Liu, K. Liu, J. Zhou. A proof of a conjecture of Mariño-Vafa on Hodge Integrals, J. Differential Geom. 65 (2003), 289-340.

[7] C.-C. Liu, K. Liu, J. Zhou. Mariño-Vafa formula and Hodge integral identities, J.Algebric Geom. 15 (2006), 379-398.

[8] K. Liu, H. Xu. Estimate of denominators of Hodge integrals, preprint, 2006.

[9] W. Lu. A note of the Mariño-Vafa formula, Science in China Ser. A Math. (11) 35 (2005), 1276-1287. (in chinese)

[10] M. Mariño, C. Vafa. Framed knots at large $N$, Orbifolds in mathematics and Physics (Madison, WI, 2001), 185-204, Contemp. Math., 310, Amer. Math. Soc., Providence, RI, 2002.

[11] D. Mumford. Towards an enumerative geometry of the moduli space of curves, in Arithmetric and geometry, Vol, II, Progr. Math., 36, Birkhuser Boston, Boston, 1983, pp. 271-328.

[12] J. Zhou. Some closed formulas and conjectures for Hodge integrals, Math. Res. Lett. 10 (2003), 275-286.

[13] _ Hodge integrals, Hurwitz numbers, and symmetric group, preprint: math.AG/0308024.

Center of Mathematical Sciences, Zhejiang University, Hangzhou, 310027, China

E-mail address: yili@cms.zju.edu.cn 